Fluctuations of conserved charges at finite temperature from lattice QCD

This article has been downloaded from IOPscience. Please scroll down to see the full text article.

2013 J. Phys.: Conf. Ser. 432012012

(http://iopscience.iop.org/1742-6596/432/1/012012)

View the table of contents for this issue, or go to the journal homepage for more

Download details:

IP Address: 134.94.122.242

The article was downloaded on $27 / 06 / 2013$ at $14: 11$

Please note that terms and conditions apply. 


\title{
Fluctuations of conserved charges at finite temperature from lattice QCD
}

\author{
S. Krieg ${ }^{1,2}$, for the Wuppertal-Budapest collaboration \\ S. Borsanyi ${ }^{1}$, Z. Fodor ${ }^{1,2,3}$, S. D. Katz ${ }^{1,3}$, C. Ratti ${ }^{4}$, K. K. Szabo ${ }^{1}$ \\ ${ }^{1}$ Fachbereich C - Physik, Bergische Universität Wuppertal, D-42119 Wuppertal, Germany \\ 2 IAS, JSC, Forschungszentrum Jülich GmbH, D-52425 Jülich, Germany \\ ${ }^{3}$ Institute for Theoretical Physics, Eötvös University, H-1117 Budapest, Hungary \\ ${ }^{4}$ Università degli Studi di Torino and INFN, Sezione di Torino, I-10125 Torino, Italy \\ E-mail: s.krieg@fz-juelich.de
}

\begin{abstract}
Recent results on fluctuations of conserved charges are reviewed and compared between different collaborations, where good agreement is found. Uncertainties of the lattice QCD simulations involved are discussed. Dedicated simulations addressing some of these uncertainties are shown, which indicate that, in the parameter regions studied so far, they are under control.
\end{abstract}

\section{Introduction}

The Quantum Chromodynamics (QCD) phase diagram is presently studied at various ultrarelativistic heavy-ion experiments, such as CERN SPS, RHIC at Brookhaven National Laboratory, ALICE at the LHC, and, in the future, FAIR at GSI, Darmstadt. One particular subject of these studies is the QCD transition between the hot quark-gluon-plasma to the hadronic 'phase', which is predicted to be an analytic crossover by Lattice QCD simulations [1]. A lot of effort is invested to find observables that would signal the QCD phase transition in an unambiguous way. Correlations and fluctuations of conserved charges have been proposed for this purpose $[2,3]$, since these quantities have significantly different values in a confined or a deconfined system.

Fluctuations of conserved charges can be obtained as linear combinations of diagonal and nondiagonal quark number susceptibilities, which can be calculated on the lattice at zero chemical potential $[4,5]$. These observables can give us an insight into the nature of the matter under study $[4,6]$. Diagonal susceptibilities measure the response of the quark number density to changes in the chemical potential, and show a rapid rise in the vicinity of the phase transition. Non-diagonal susceptibilities give us information about the correlation between different flavors. They are supposed to vanish in a non-interacting quark-gluon plasma (QGP). It was shown in Ref. [7] that correlations between different flavors are nonzero in perturbative QCD at large temperatures due to the presence of flavor-mixing diagrams: a quantitative analysis of this observable allows one to draw conclusions about the presence of bound states in the QGP [8]. Another observable which was proposed to this purpose, and which can be obtained from a combination of diagonal and non-diagonal quark number susceptibilities, is the baryonstrangeness correlator [9]. 

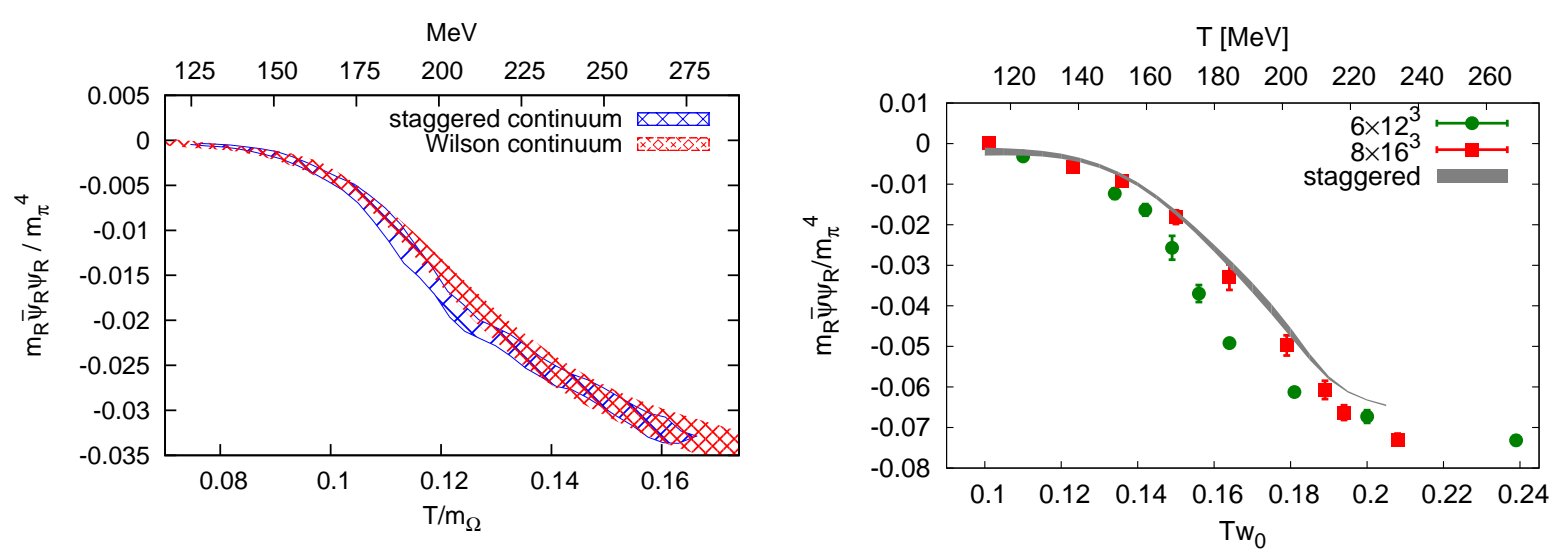

Figure 1. Crosschecks between different lattice actions. Left panel: The continuum extrapolated chiral condensate obtained from simulations with $N_{f}=2+1$ flavors of quarks at a pion mass of $545 \mathrm{MeV}$, using Wilson and staggered fermions [11]. Right panel: The continuum extrapolated chiral condensate obtained from simulations with $N_{f}=2$ flavors of quarks at a pion mass of $350 \mathrm{MeV}$, using overlap and staggered quarks [12].

In the present contribution we will first discuss uncertainties of lattice QCD simulations and show results from dedicated simulations providing cross-checks for our calculations. We will then discuss continuum extrapolated results of our collaboration on some of the aforementioned observables. We compare our results to the predictions of the HRG model with resonances up to $2.5 \mathrm{GeV}$ mass at small temperatures, and of the Hard Thermal Loop (HTL) resummation scheme at large temperatures, where available. Furthermore, we will compare our results to those of the hotqcd collaboration.

In our simulations we use $N_{f}=2+1$ dynamical staggered quark flavors, in a temperature regime between 125 and $400 \mathrm{MeV}[10]$ and set the light and strange quark masses to their physical values. Lattices with $N_{t}=6,8,10,12$ are used. Continuum extrapolations are performed for all observables under study. Further details can be found in [10].

\section{Discussion of uncertainties}

Simulations in finite temperature Lattice QCD typically use the staggered formalism. In this formalism one is faced with the problem that the Dirac operator does not implement a single quark flavor, but rather four so-called "tastes", which correspond to four flavors in the continuum limit. The tastes' degeneracy is lifted by non-physical (continuum irrelevant) so-called tastebreaking interactions. As a consequence, the pion sector is distorted and contains, in addition to the one would-be Goldstone-boson, 15 additional "pions". This results in an rms pion mass which is larger than that of the "real" pion. The impact of this effect on the thermodynamic observables has been recently discussed in the HRG framework [13, 14].

Improved action reduce the taste-breaking effects considerably, mostly by using "smearing", i.e. by averaging over gauge links aiming to reduce ("UV" related) lattice artifacts. While this procedure is very successful at taming the distorted pion sector, one still has to deal with the fact that one is simulating more flavors than one actually wants. This is addressed by the so-called "rooting trick" in which the fermionic determinant is taken to be an appropriate root (e.g. square root or fourth root) of the staggered determinant. Accumulating practical evidence suggests that this is a viable procedure, but, as of today, a proof of its correctness is still missing.

One way to check for problems related to these above issues is to repeat simulations with other lattice actions that are free of such uncertainties and to compare the results. Ideally, 


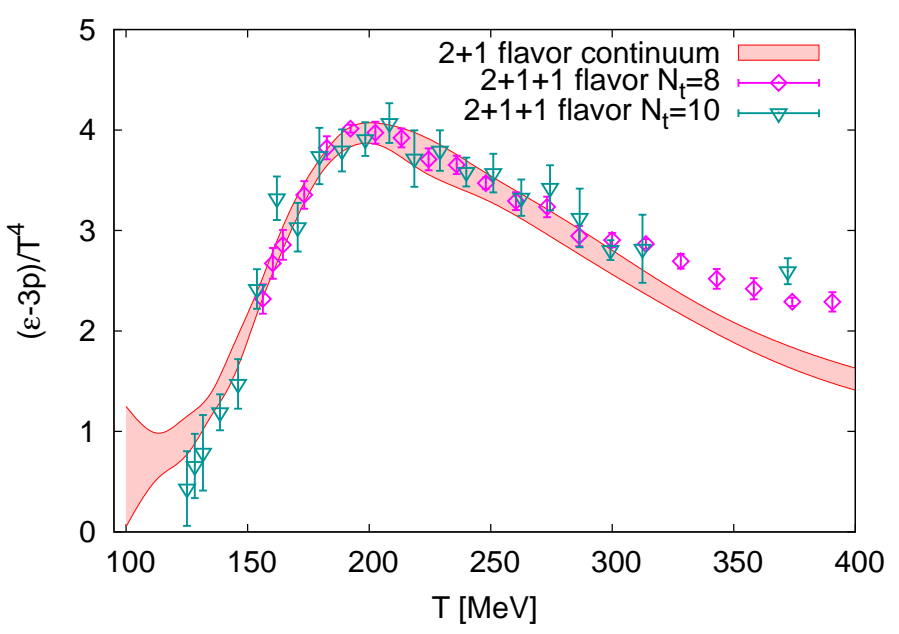

Figure 2. $N_{F}=2+1$ Equation of State of QCD compared with individual data points obtained with an additional dynamical charm $\left(N_{f}=2+1+1\right)$. At temperatures above $\approx 300 \mathrm{MeV}$ the charm quark becomes relevant.

one would want to do that for all results, if it were not for the computational costs this does require. Therefore, a reasonable first step is to perform cross-checks at some parameter values, such as with larger pion masses (and thus at lower costs), but still in the continuum limit. Such a calculation using Wilson fermions is shown on the left panel of Fig. 1. Here, simulations were performed at a pion mass of about $545 \mathrm{MeV}$ and a direct comparison for the continuum extrapolated chiral condensate is shown (Details on the simulation and on the renormalization procedure used can be found in [11]). The results agree within errors. Another, albeit not continuum extrapolated, comparison for the same observable is made on the right panel of Fig. 1, where the staggered results are compared to ones obtained with overlap fermions. Here, the pion mass is even lower. Since the continuum limit is still missing, one cannot make any final statements, however, the nice agreement of the fine data set $\left(8 \times 16^{3}\right)$ with the staggered continuum estimate suggest that also here, agreement between the different formulations can be expected (the coarse data set is likely not in the scaling region). Note, that both the staggered and overlap simulations in this latter comparison did not include a dynamical strange quark. Since here we only want to perform a cross-check of the staggered formalism, this is also not required. Related to this latter comment, for some quantities at temperatures above ca. $300 \mathrm{MeV}$, certainly for Equation of State of QCD, a dynamical charm quark is required, as Fig. 2 shows. This is, however, most likely not the case for fluctuations, as shown in Fig. 3 [15].

\section{Fluctuations}

\subsection{Details of the lattice simulations}

The lattice action used in our calculations is the same one we used in [16, 17], namely a tree-level Symanzik improved gauge, and a stout-improved staggered fermion action (see Ref. [18] for details). The stout-smearing procedure [19], as mentioned earlier, yields an improved discretization of the fermion-gauge vertex and reduces taste breaking artifacts. Analogously to our action, using an alternative link-smearing scheme, the HISQ action [20] suppresses the taste breaking in a similar way. The latter is used by the hotQCD collaboration in its latest studies $[21,22,23]$. For details about the simulation algorithm we refer the reader to [17].

In analogy with what we did in $[16,17]$, we set the scale at the physical point by simulating 

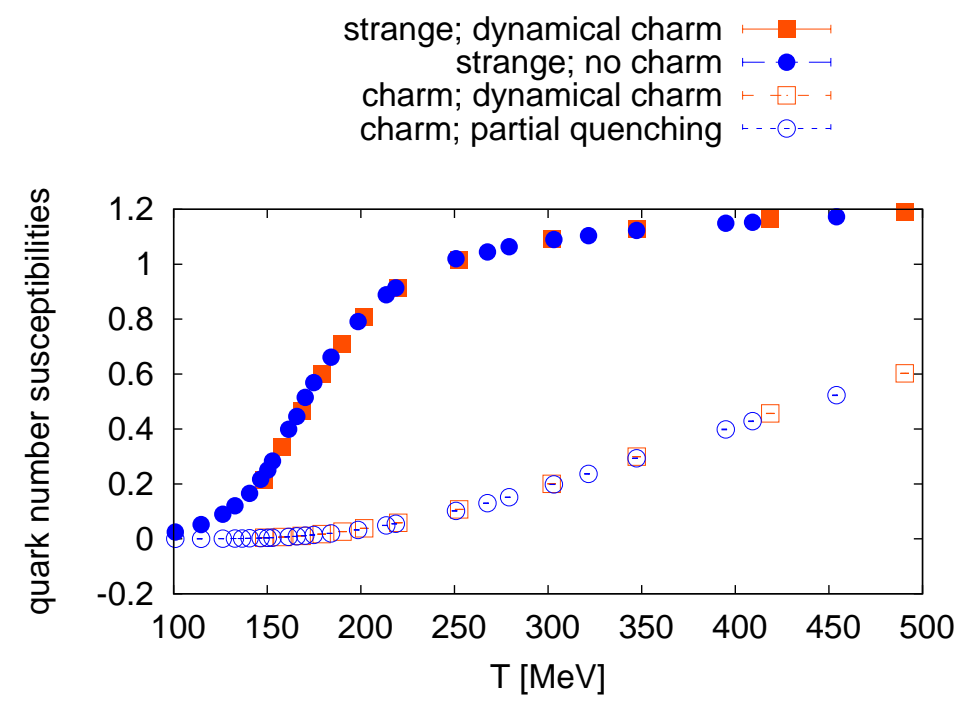

Figure 3. Strange and charm susceptibilities with and without dynamical charm.

at $T=0$ with physical quark masses [17] and reproducing the kaon and pion masses and the kaon decay constant. This gives an uncertainty of about $2 \%$ in the scale setting.

\subsection{Observables under study}

The baryon number $B$, strangeness $S$ and electric charge $Q$ fluctuations can be obtained, at vanishing chemical potentials, from the QCD partition function. The relationships between the quark chemical potentials and those of the conserved charges are as follows:

$$
\begin{aligned}
\mu_{u} & =\frac{1}{3} \mu_{B}+\frac{2}{3} \mu_{Q} \\
\mu_{d} & =\frac{1}{3} \mu_{B}-\frac{1}{3} \mu_{Q} \\
\mu_{s} & =\frac{1}{3} \mu_{B}-\frac{1}{3} \mu_{Q}-\mu_{S} .
\end{aligned}
$$

Starting from the QCD pressure,

$$
\frac{p}{T^{4}}=\frac{1}{V T^{3}} \ln Z\left(V, T, \mu_{B}, \mu_{S}, \mu_{Q}\right)
$$

we can define the moments of charge fluctuations as follows:

$$
\chi_{l m n}^{B S Q}=\frac{\partial^{l+m+n} p / T^{4}}{\partial\left(\mu_{B} / T\right)^{l} \partial\left(\mu_{S} / T\right)^{m} \partial\left(\mu_{Q} / T\right)^{n}} .
$$

We will concentrate on the quadratic fluctuations

$$
\chi_{2}^{X}=\frac{1}{V T^{3}}\left\langle N_{X}^{2}\right\rangle
$$

and on the correlators among different charges or quark flavors:

$$
\chi_{11}^{X Y}=\frac{1}{V T^{3}}\left\langle N_{X} N_{Y}\right\rangle .
$$


Given the relationships between chemical potentials (1) the diagonal susceptibilities of the conserved charges can be obtained from quark number susceptibilities in the following way:

$$
\begin{aligned}
\chi_{2}^{B} & =\frac{1}{9}\left[\chi_{2}^{u}+\chi_{2}^{d}+\chi_{2}^{s}+2 \chi_{11}^{u s}+2 \chi_{11}^{d s}+2 \chi_{11}^{u d}\right] \\
\chi_{2}^{Q} & =\frac{1}{9}\left[4 \chi_{2}^{u}+\chi_{2}^{d}+\chi_{2}^{s}-4 \chi_{11}^{u s}+2 \chi_{11}^{d s}-4 \chi_{11}^{u d}\right] \\
\chi_{2}^{I} & =\frac{1}{4}\left[\chi_{2}^{u}+\chi_{2}^{d}-2 \chi_{11}^{u d}\right] \\
\chi_{2}^{S} & =\chi_{2}^{s} .
\end{aligned}
$$

If we do not wish to take further derivatives, we can take all three chemical potentials $(u, d, s)$ to zero. In this case, nothing distinguishes between the $u$ and $d$ derivative: this gives slightly simplified formulae:

$$
\begin{aligned}
\chi_{2}^{B} & =\frac{1}{9}\left[2 \chi_{2}^{u}+\chi_{2}^{s}+4 \chi_{11}^{u s}+2 \chi_{11}^{u d}\right] \\
\chi_{2}^{Q} & =\frac{1}{9}\left[5 \chi_{2}^{u}+\chi_{2}^{s}-2 \chi_{11}^{u s}-4 \chi_{11}^{u d}\right] \\
\chi_{2}^{I} & =\frac{1}{2}\left[\chi_{2}^{u}-\chi_{11}^{u d}\right] .
\end{aligned}
$$

The baryon-strangeness correlator, which was proposed in Ref. [9] as a diagnostic to understand the nature of the degrees of freedom in the QGP, has the following expression in terms of quark number susceptibilities:

$$
C_{B S}=-3 \frac{\left\langle N_{B} N_{S}\right\rangle}{\left\langle N_{S}^{2}\right\rangle}=1+\frac{\chi_{11}^{u s}+\chi_{11}^{d s}}{\chi_{2}^{s}} .
$$

\subsection{Results}

The first observables we discuss are the diagonal light and strange quark number susceptibilities: their behavior as functions of the temperature is shown in the two panels of Fig. 4. The different symbols correspond to different values of $N_{t}$, from 8 to 16 . The red band is the continuum extrapolation, obtained from the unimproved data, not from the improved ones shown in the figure. The continuum extrapolation is performed through a parabolic fit in the variable $\left(1 / N_{t}\right)^{2}$, over five $N_{t}$ values from 6 to 16 . The band shows the spread of the results of other possible fits. The comparison between the improved data and the continuum bands in the figure shows the success of the improvement program throughout the entire temperature range. Both observables show a rapid rise in a certain temperature range, and reach approximately $90 \%$ of the ideal gas value at large temperatures. However, the temperature around which the susceptibilities rise is approximately 15-20 MeV larger for strange quarks than for light quarks. Besides, the light quark susceptibility shows a steeper rise with temperature, compared to the strange quark one. They approach each other at high temperatures. The pattern of temperature dependence is strongly related to the actual quark mass. The difference between the light and strange susceptibilities computed at the physical pion mass is more pronounced than in earlier works with heavier pion masses [24, 25]. This is more evident in Fig. 5: in the left panel we show the continuum extrapolation of both susceptibilities on the same plot. In the right panel we show the ratio $\chi_{s} / \chi_{u}$ : it reaches 1 only around $300 \mathrm{MeV}$, while for smaller temperatures it is $<1$. It is worth noticing that all these observables agree with the corresponding HRG model predictions for temperatures below the transition. 

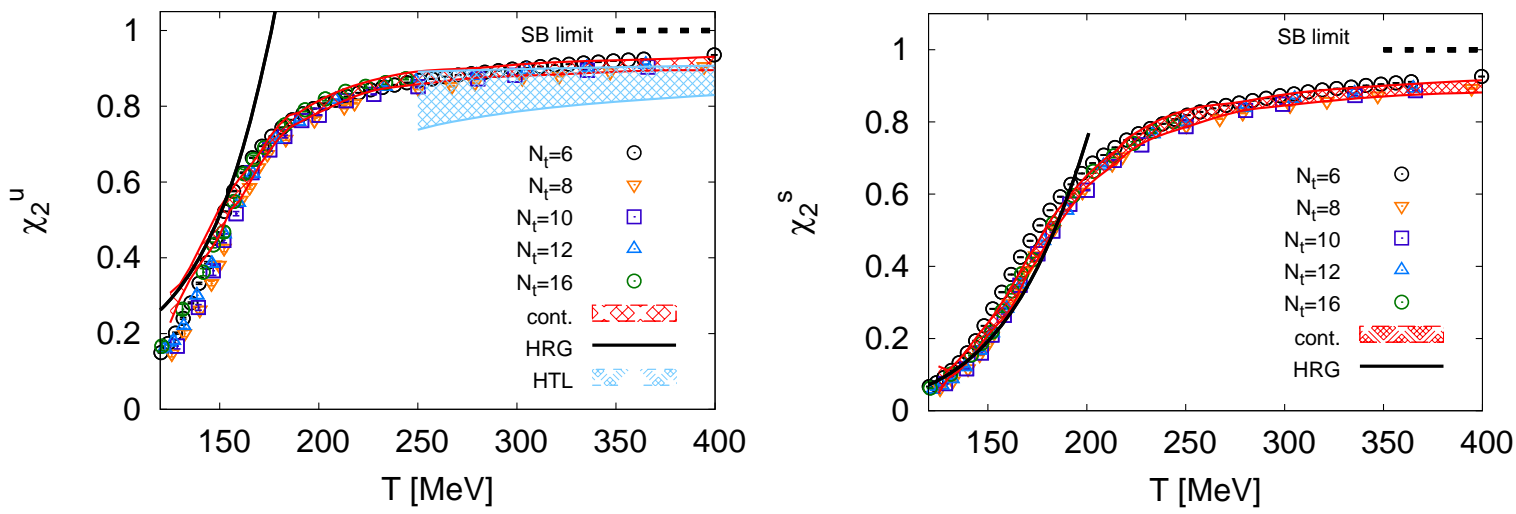

Figure 4. Left panel: diagonal light quark susceptibility as a function of the temperature. Right panel: diagonal strange quark susceptibility as a function of the temperature. In both panels, the different symbols correspond to different $N_{t}$ values. The red band is the continuum extrapolation. The black curve is the HRG model prediction for these observables. The dashed line shows the ideal gas limit. The light blue band in the left panel is the HTL prediction taken from Ref. [7].
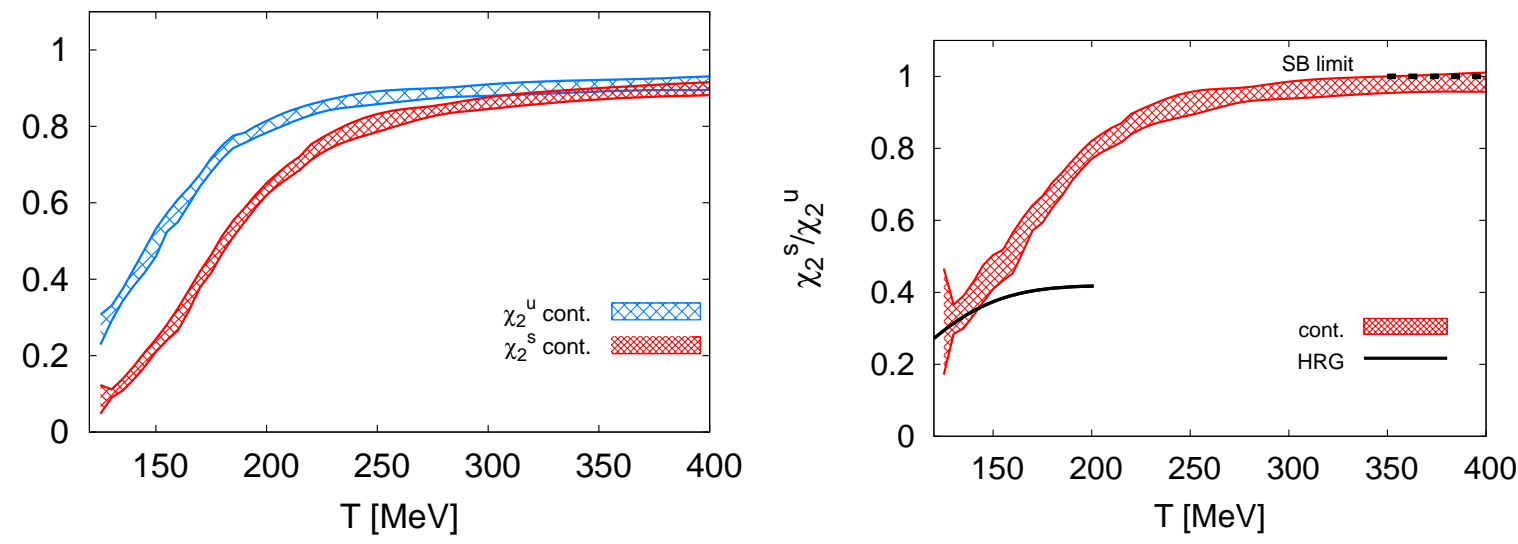

Figure 5. Left panel: direct comparison between the continuum limit of light and strange quark susceptibilities. Right panel: ratio $\chi_{2}^{s} / \chi_{2}^{u}$ as a function of the temperature. The red band is the lattice continuum result. The black, solid curve is the HRG model prediction. The dashed line indicates the ideal gas limit.

The non-diagonal us susceptibility measures the degree of correlation between different flavors. This observable vanishes in the limit of an ideal, non-interacting QGP. However, flavormixing diagrams in perturbative QCD yield a finite value for this correlation also at large temperatures [7]. We show our result in Fig. 6. $\chi_{11}^{u s}$ is non-zero in the entire temperature range under study. It has a dip in the vicinity of the transition, where the correlation between $u$ and $s$ quarks turns out to be maximal. It agrees with the HRG model prediction in the hadronic phase. This correlation stays finite and large for a certain temperature range above the transition temperature $T_{c}[16,17,14]$. A quantitative comparison between lattice results and predictions for a purely partonic QGP state can give us information about the probability of bound states survival above $T_{c}[8]$. 


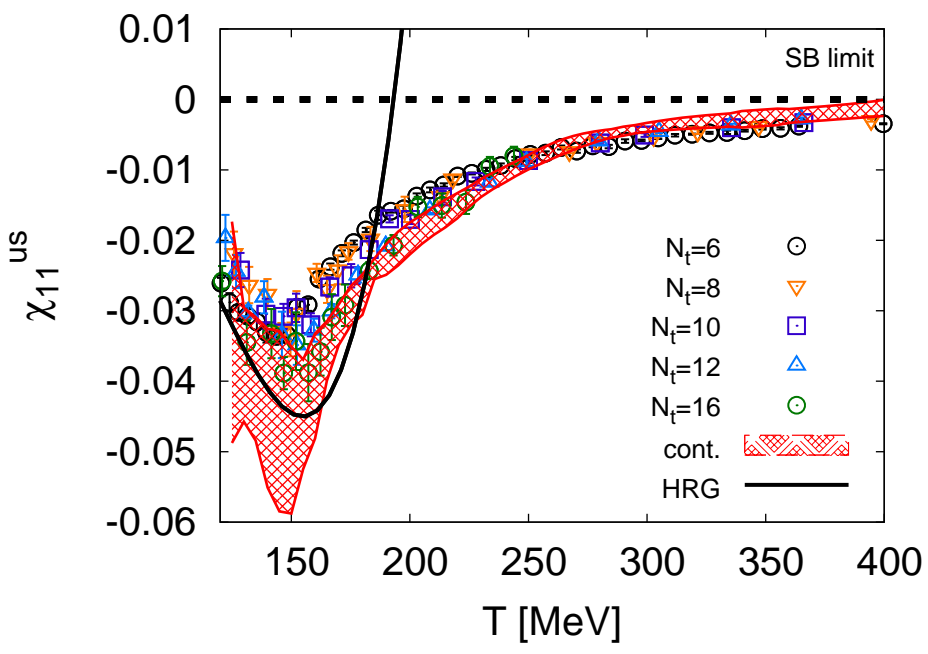

Figure 6. Non-diagonal u-s correlator as a function of the temperature. The different symbols correspond to different $N_{t}$ values. The red curve is the continuum extrapolated result. The black curve is the HRG model prediction. The dashed line indicates the ideal gas limit for this observable.

Quadratic baryon number, electric charge and isospin fluctuations can be obtained from the above partonic susceptibilities through Eqs. (7). We show our results for these observables in Fig. 7 and in the left panel of Fig. 8. In the low-temperature, hadronic phase we have a very good agreement with the HRG model predictions. In the vicinity of the phase transition, these quantities all show a rapid rise with temperature, in analogy with what already observed for the light and strange quark susceptibilities. At large temperature they reach approximately $90 \%$ of their respective ideal gas values. A comparison between all diagonal susceptibilities, rescaled by their corresponding Stefan-Boltzmann limits, is shown in the right panel of Fig. 8, from which it is evident that they all show similar features in their temperature dependence, even if the temperature at which they rise is larger for the strangeness and baryon number susceptibilities.

The baryon-strangeness correlator $C_{B S}$ defined in Eq. (8) was proposed long ago [9] as a diagnostic for strongly interacting matter. It is supposed to be equal to one for a non-interacting QGP, while it is temperature-dependent and generally smaller than one in a hadronic system. We show our result for this observable in Fig. 9. At the smallest temperatures it agrees with the HRG model result, and it shows a rapid rise across the phase transition. It reaches the ideal gas limit much faster than the other observables under study, yet there is a window of about $100 \mathrm{MeV}$ above $T_{c}$, where its value is still smaller than one. In analogy with $\chi_{11}^{u s}$, this observable seems to leave some room for bound states survival above $T_{c}$.

In Fig. 10, finally, we compare our results to those of the hotQCD collaboration. In the temperature region, were both collaborations have data, the results agree within errors.

\section{Conclusions}

We have discussed uncertainties of Lattice QCD simulations and have presented continuum results of our collaboration on diagonal and non-diagonal quark number susceptibilities. Our results were obtained simulating $N_{F}=2+1$ dynamical staggered quark flavors with physical masses, in a temperature range between 125 and $400 \mathrm{MeV}$. The continuum extrapolations were based on $N_{t}=6,8,10,12$ and 16 lattices. We calculated the systematic errors by varying over the ambiguities of the possible extrapolations. 

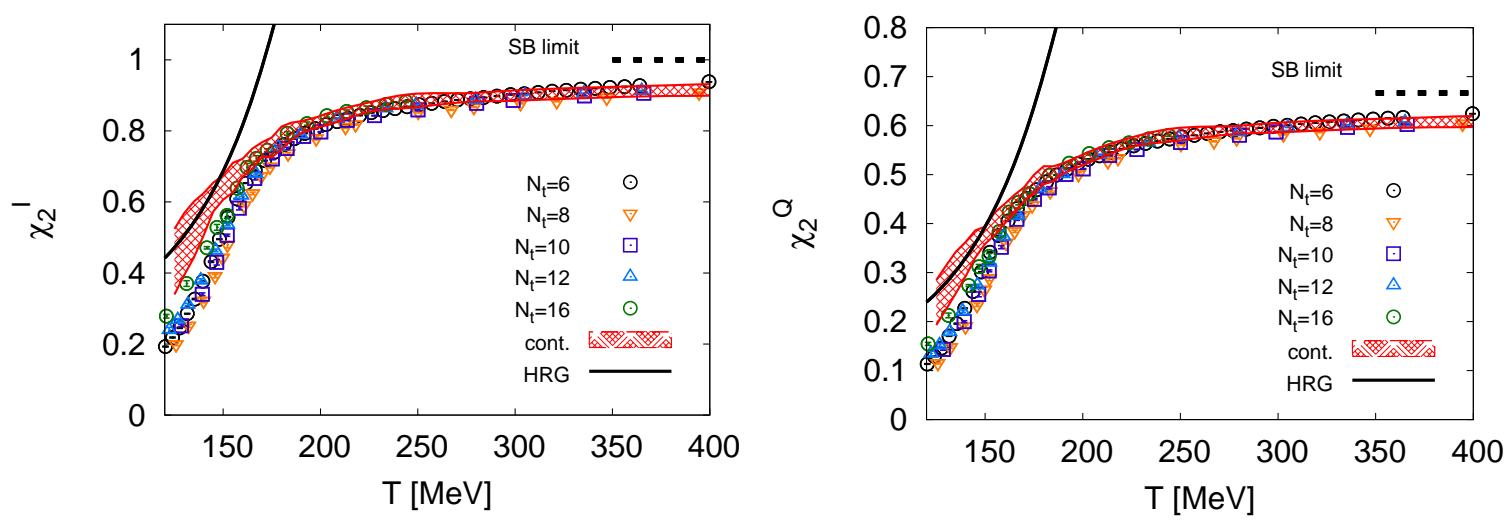

Figure 7. Left panel: isospin susceptibility as a function of the temperature. Right panel: electric charge susceptibility as a function of the temperature. In both panels, the different dots correspond to different $N_{t}$ values. The red band is the continuum extrapolation. The black curve is the HRG model prediction for these observables. The dashed line shows the ideal gas limit.
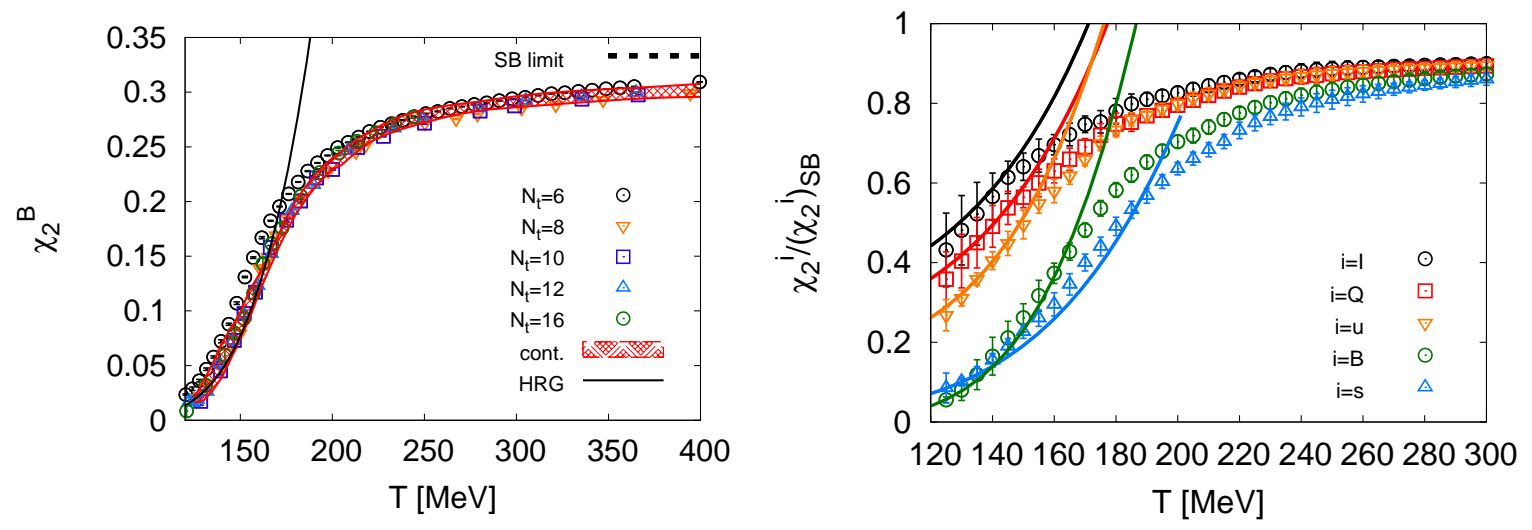

Figure 8. Left panel: quadratic fluctuation of baryon number as a function of the temperature. The different symbols correspond to different $N_{t}$ values, the red band is the continuum extrapolation and the black, solid curve is the HRG model result. The ideal gas limit is shown by the black, dashed line. Right panel: comparison between all diagonal susceptibilities, rescaled by the corresponding ideal gas limit, as functions of the temperature.

All observables consistently show a very good agreement with the HRG model predictions for temperatures below the phase transition.

The diagonal fluctuations have some common features: they all show a rapid rise in the vicinity of the phase transition, and reach approximately $90 \%$ of the corresponding ideal gas value at large temperatures. The rise of both strange quark and baryon number susceptibilities is shifted to temperatures about $20 \mathrm{MeV}$ higher than those for light quark, charge and isospin susceptibilities. Non-diagonal flavor and charge correlators remain different from their ideal gas values for a certain window of temperatures above the transition, thus not excluding the possibility of bound state survival above $T_{c}$. We have compared our results to those by the hotQCD collaboration and found them to be in agreement within errors. 


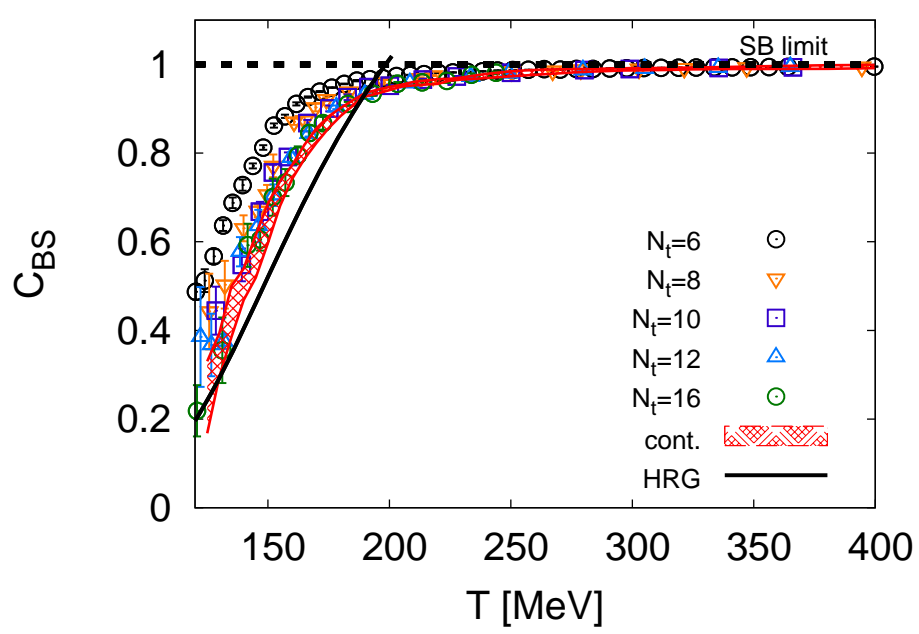

Figure 9. Baryon-strangeness correlator as a function of the temperature. The different symbols correspond to different $N_{t}$ values, the red band is the continuum extrapolation and the black, solid curve is the HRG model result. The ideal gas limit is shown by the black, dashed line.
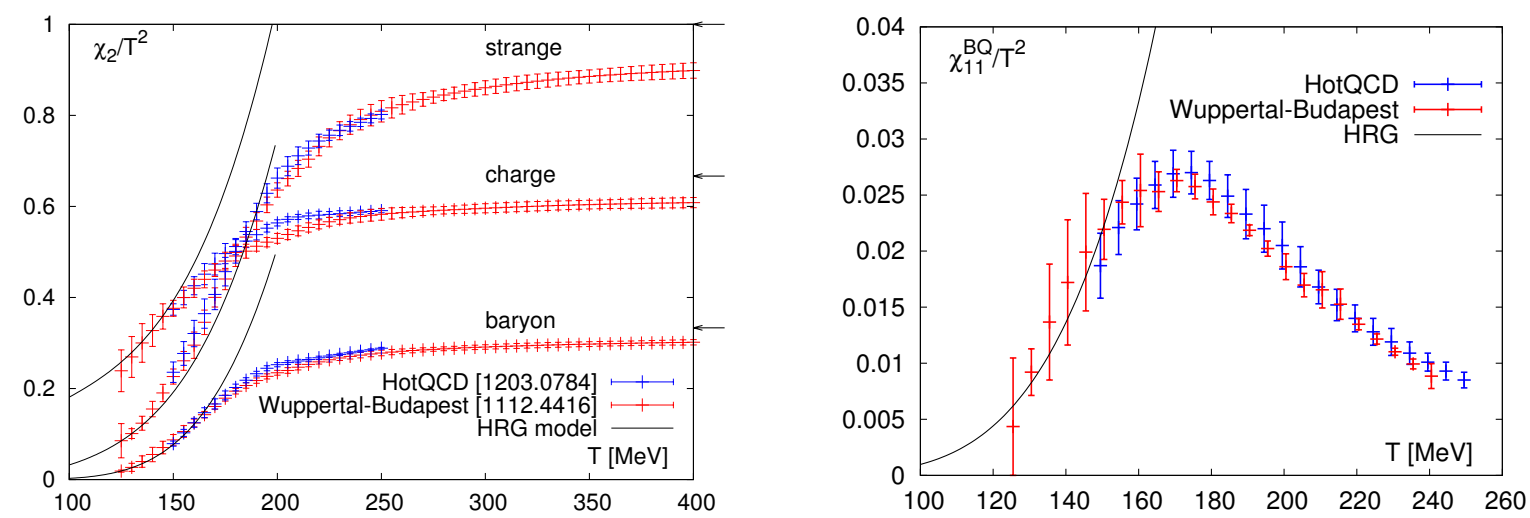

Figure 10. Diagonal and off-diagonal correlators of conserved charges as a function of temperature. Only continuum extrapolated lattice data are shown. There is a good agreement between hotQCD [26] and Wuppertal-Budapest [10] data, obtained using the HISQ and stout actions, respectively. The broader temperature region by the Wuppertal-Budapest collaboration allows a comparison with the HRG model's prediciton, and was used to benchmark resummation schemes at high temperature [27].

\section{Acknowledgments}

Computations were carried out at the Universities Wuppertal and Budapest on GPU clusters [28], on the Wuppertal QPACE machine and at Forschungszentrum Juelich. This work is supported in part by the Deutsche Forschungsgemeinschaft grants FO 502/2 and SFB- TR 55, by the EU (FP7/2007-2013)/ERC no. 208740 and by the Italian Ministry of Education, Universities and Research under the Firb Research Grant RBFR0814TT.

\section{References}

[1] Aoki Y, Endrodi G, Fodor Z, Katz S and Szabo K 2006 Nature 443 675-678 (Preprint hep-lat/0611014) 
[2] Asakawa M, Heinz U W and Muller B 2000 Phys.Rev.Lett. 85 2072-2075 (Preprint hep-ph/0003169)

[3] Jeon S and Koch V 2000 Phys.Rev.Lett. 85 2076-2079 (Preprint hep-ph/0003168)

[4] Gottlieb S A, Liu W, Toussaint D, Renken R and Sugar R 1988 Phys.Rev. D38 2888-2896

[5] Gavai R, Potvin J and Sanielevici S 1989 Phys.Rev. D40 2743

[6] McLerran L D 1987 Phys.Rev. D36 3291

[7] Blaizot J, Iancu E and Rebhan A 2001 Phys.Lett. B523 143-150 (Preprint hep-ph/0110369)

[8] Ratti C, Bellwied R, Cristoforetti M and Barbaro M 2012 Phys.Rev. D85 014004 (Preprint 1109.6243)

[9] Koch V, Majumder A and Randrup J 2005 Phys.Rev.Lett. 95182301 (Preprint nucl-th/0505052)

[10] Borsanyi S, Fodor Z, Katz S D, Krieg S, Ratti C et al. 2012 JHEP 1201138 (Preprint 1112.4416)

[11] Borsanyi S, Durr S, Fodor Z, Hoelbling C, Katz S D et al. 2012 JHEP 1208126 (Preprint 1205.0440)

[12] Borsanyi S, Delgado Y, Durr S, Fodor Z, Katz S D et al. 2012 Phys.Lett. B713 342-346 (Preprint 1204.4089)

[13] Huovinen P and Petreczky P 2010 Nucl.Phys. A837 26-53 (Preprint 0912.2541)

[14] Borsanyi S et al. (Wuppertal-Budapest Collaboration) 2010 JHEP 1009073 (Preprint 1005.3508)

[15] Borsanyi S, Endrodi G, Fodor Z, Katz S D, Krieg S et al. 2011 PoS LATTICE2011 201 (Preprint 1204. 0995)

[16] Aoki Y, Fodor Z, Katz S and Szabo K 2006 Phys.Lett. B643 46-54 (Preprint hep-lat/0609068)

[17] Aoki Y, Borsanyi S, Durr S, Fodor Z, Katz S D et al. 2009 JHEP 0906088 (Preprint 0903.4155 )

[18] Aoki Y, Fodor Z, Katz S and Szabo K 2006 JHEP 0601089 (Preprint hep-lat/0510084)

[19] Morningstar C and Peardon M J 2004 Phys.Rev. D69 054501 (Preprint hep-lat/0311018)

[20] Follana E et al. (HPQCD Collaboration, UKQCD Collaboration) 2007 Phys.Rev. D75 054502 (Preprint hep-lat/0610092)

[21] Bazavov A and Petreczky P (HotQCD Collaboration) 2009 PoS LAT2009 163 (Preprint 0912.5421)

[22] Bazavov A and Petreczky P (HotQCD collaboration) 2010 J.Phys.Conf.Ser. 230012014 (Preprint 1005.1131)

[23] Bazavov A, Bhattacharya T, Cheng M, DeTar C, Ding H et al. 2012 Phys.Rev. D85 054503 (Preprint 1111.1710)

[24] Gavai R and Gupta S 2006 Phys.Rev. D73 014004 (Preprint hep-lat/0510044)

[25] Cheng M, Hendge P, Jung C, Karsch F, Kaczmarek O et al. 2009 Phys.Rev. D79 074505 (Preprint 0811.1006)

[26] Bazavov A et al. (HotQCD Collaboration) 2012 Phys.Rev. D86 034509 (Preprint 1203.0784)

[27] Andersen J O, Mogliacci S, Su N and Vuorinen A 2012 (Preprint 1210.0912)

[28] Egri G I, Fodor Z, Hoelbling C, Katz S D, Nogradi D et al. 2007 Comput.Phys.Commun. 177 631-639 (Preprint hep-lat/0611022) 